\title{
Resonant Transmission Through a Single Subwavelength Slit for Varied Metallic Permittivities and Its Modal Orthogonality
}

\author{
Jong-Eon Park ${ }^{1}{ }^{\circledR}$, Hosung Choo $^{2}$ and Young-Ki Cho ${ }^{3, *}$ \\ 1 Metamaterial Electronic Device Research Center, Hongik University, Seoul 04066, Korea; \\ jongeon.park@gmail.com \\ 2 School of Electronic and Electrical Engineering, Hongik University, Seoul 04066, Korea; hschoo@hongik.ac.kr \\ 3 School of Electronics Engineering, Kyungpook National University, Daegu 41566, Korea \\ * Correspondence: ykcho@ee.knu.ac.kr
}

Received: 5 December 2019; Accepted: 14 January 2020; Published: 16 January 2020

Featured Application: The results obtained in this work provide a useful set of benchmark data to interpret experimental results and to inform design efforts of slit-based structures in the terahertz range. The present method of analysis can also be applicable to problems involving multiple slits with different widths.

\begin{abstract}
This article investigates resonant transmission phenomena through a single metallic subwavelength slit when the permittivity of a real metal varies. The single metallic slit is utilized as a metal-insulator-metal waveguide, and a mode-matching technique is employed to obtain the transmitted power. The periodic resonant transmission phenomena (in terms of the metallic plate thickness) are solved, and the resonances can be understood by their guide wavelengths. Even when the permittivity of the real metal includes imaginary parts (i.e., metal with loss), the resonant transmittances are obtained. However, the peaks of the transmittances decrease, as the plate thickness increases. The orthogonal relationship of an incomplete orthogonal set is maintained despite metallic loss (given a relatively small amount of loss), due to the complex permittivity of the real metal.
\end{abstract}

Keywords: transmission cross-section; metal-insulator-metal waveguide; mode-matching technique

\section{Introduction}

Electromagnetic resonant transmission through one- or two-dimensional slits has been discussed for several decades, and research on extraordinary optical transmission [1-4] has led to significant physical and practical achievements. In earlier research [5,6], perforated metallic plates are generally assumed to be perfect electric conductors (PECs), and corresponding resonant transmission characteristics through PEC slits were investigated. However, studies were limited to the microwave or millimeter-wave regimes and did not extend to the terahertz (THz) range. Therefore, it remains important to investigate resonant transmission phenomena through real metallic slits by considering actual permittivities of metals, when the frequency goes beyond the microwave range. To extend transmittance study to the $\mathrm{THz}$ range, the geometry of a metal-insulator-metal (MIM) waveguide was adopted, and the resulting modes formed in the MIM waveguide were examined. The resonant transmission phenomena were then systematically analyzed through a real metallic slit in the THz range [7-9]. Thanks to this fundamental research, resonant transmission phenomena have been investigated at wide $\mathrm{THz}$ frequencies by taking into account actual permittivities for various real metals $[10,11]$ and discussing transmittances through periodic metallic slits [12]. These studies found that transmittance phenomena through metallic slits 
are similar to those in PEC cases at lower frequencies. However, large discrepancies are apparent at higher $\mathrm{THz}$ frequencies [10].

Slit transmittances in wide $\mathrm{THz}$ ranges can usually be solved easily via the finite-difference time-domain (FDTD) or finite element method $[13,14]$ and etc. $[15,16]$. While transmittance results can be obtained directly under those methods, their physical meanings are not easily understood using such "brute-force" numerical techniques. Brute-force numerical techniques require substantial numbers of mesh grids, causing corresponding numerical dispersion errors to increase gradually at higher frequencies [14]. By contrast, the mode-matching technique (MMT) does not require grids and, consequently, has no dispersion errors. The MMT also provides physical meanings by obtaining the guide wavelengths of an MIM waveguide, making it easy to understand the resonant transmittance as a function of the metallic plate thickness. Once the eigenvalues for the geometry are obtained herein, then the transmittances as a function of the plate thickness can be resolved easily.

Since electromagnetic waves do not penetrate into a PEC, no mode in the PEC region needs to be accounted for. For actual metallic plates, however, modes in the metallic region should be considered, because a tiny amount of each mode permeates into the metallic region due to their finite conductivities. To be precise, modal components in the metallic region (as well as the slit region) should be fully considered when solving transmittances. In addition, for the finite conductivity of a metal, orthogonal properties are not completely satisfied, while the orthogonality for the PEC MIM waveguide is completely fulfilled. Therefore, a validation issue of slit transmittances could be raised for real metallic slits in accordance with orthogonality.

In this research, the procedure obtaining the transmittances by the MMT is as follows. First, the modes constituting each region in the free space and an MIM waveguide need to be obtained in advance. To calculate modal eigenvalues in the MIM waveguide, a dispersion equation should be applied [9]. Then, tangential incident, reflected, and transmitted electromagnetic fields are set to be continuous at each junction. The unknowns in the electromagnetic field equations can be solved though the system of equations, and the reflection and transmission coefficients can be determined. The overall reflected and transmitted electromagnetic fields of a single-slit geometry can be obtained by using multiregion problems. The entire computing procedure is listed and explained in detail in [10].

The results using the MMT in this paper have several unique features compared to previous studies in the following aspects. First, resonant transmittance patterns are studied in detail as a function of the permittivity of a real metal, when the metal is lossy. Then, periodic transmission peaks and attenuations are examined, as the thickness of the plate increases. We also observe changes in guide wavelengths and resonant transmittances, as the imaginary part of the permittivity of the metal decreases, which cannot be obtained by other numerical techniques, such as the FDTD method or finite element method. Finally, orthogonal relations are thoroughly studied for lossless and lossy metallic plates.

These resonant transmissions or extraordinary transmissions can be applied to practical devices like band-selective spatial filters $[17,18]$ and chemical sensors $[19,20]$. In addition, controllable dual transmissions have attracted significant attention to research societies, because these phenomena can be practically applicable to research areas such as switching, sensing, polarimetry microscopy, hyperspectral imaging, and security encryption [21-26].

In this paper, a dispersion equation applied into the MMT formulation is explained first. Two transmission quantities are subsequently introduced, before the transmittances for lossless metals are obtained. Transmission characteristics for the lossy metal case are then computed, and the results are compared with the lossless case. The modal orthogonal relation, which is an important prerequisite of the MMT, is finally discussed when considering both lossy and lossless metals.

\section{Transmission Cross-Sections (TCSs) through a Subwavelength Slit for a Lossless Conducting Plate}

Figure 1 shows the geometry of an MIM waveguide, including a gap width of $2 g$ and a metal plate thickness of $d$. In this section, $2 g$ is fixed as $0.03 \lambda_{0}$, while $d$ is a variable and the structure is constant 
along the $y$-axis. An electromagnetic plane wave with parallel polarization propagates along the $z$-axis. In the metallic region, the permittivity of the metal $\varepsilon_{r}$ is generally negative and will be varied in this work to investigate resonant transmittances. We consider only even modes, because even modes are sufficient for describing normal incidence in the MIM waveguide.

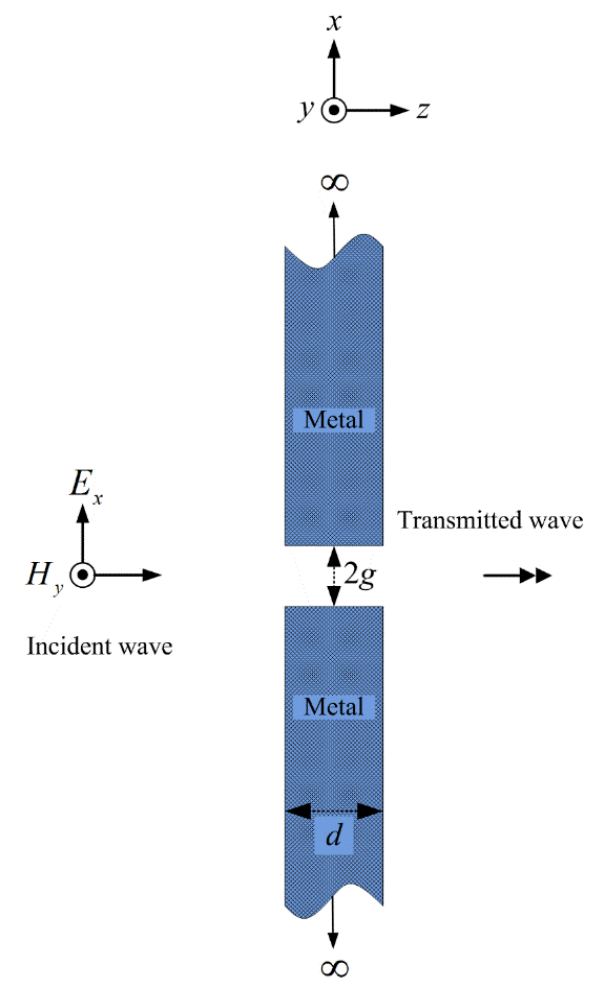

Figure 1. Geometry of a single metallic slit.

The dispersion equation is the most fundamental equation in the MIM waveguide and obtained as follows [10]:

$$
\begin{gathered}
\tanh \left(\kappa_{i, n} g\right)=-\left(\kappa_{m, n} \times \varepsilon_{r, i}\right) /\left(\kappa_{i, n} \times \varepsilon_{r, m}\right), \\
\text { where }\left(k_{z, n}\right)^{2}=\left(\kappa_{m, n}\right)^{2}+\omega^{2} \mu \varepsilon_{r, m}=\left(\kappa_{i, n}\right)^{2}+\omega^{2} \mu \varepsilon_{r, i},
\end{gathered}
$$

where $\varepsilon_{r, m}$ and $\varepsilon_{r, i}$ are permittivities in the metal and the insulator, respectively, $\kappa_{m, n}$ and $\kappa_{i, n}$ are $n$th transverse propagation constants in the metal and the insulator, respectively, and $k_{z, n}$ is the $n$th propagation constant along the $z$-axis. The subscripts $n$ in $\kappa_{m, n}, \kappa_{i, n}$, and $k_{z, n}$ commonly indicate the $n$th modes, which are coupled to one another in Equation (2). The roots $\left\{\kappa_{m, n}\right\} \mathrm{s}$ of the dispersion equation are found using Muller's method [10] while varying the geometrical parameter of $2 g$ and the relative permittivity of $\varepsilon_{r, m}$ for the metal.

The TCS transmitted into the right free space is defined as:

$$
\operatorname{TCS}[m]=\frac{P_{t}}{W_{i}}=\frac{1}{2 W_{i}} \int_{S} \operatorname{Re}\left[\vec{E} \times \vec{H}^{*}\right] \cdot\left(\vec{a}_{z} d s\right),
$$

where $P_{t}$ and $W_{i}$ are transmitted power $(\mathrm{W})$ and incident power density $(\mathrm{W} / \mathrm{m})$, respectively. As another quantity, the transmittance into the right free space is also widely used $[6,10]$ and defined as:

$$
\tau[\text { dimensionless }]=\frac{1}{2 g} \frac{P_{t}}{W_{i}}=\frac{1}{2 g} \cdot \frac{1}{2 W_{i}} \int_{S} \operatorname{Re}\left[\vec{E} \times \vec{H}^{*}\right] \cdot\left(\vec{a}_{z} d s\right) .
$$

From Equation (3), the TCSs were obtained by the MMT and plotted in Figure 2a-d as a function of the permittivities of real metals. Figure 2a shows the TCS through a PEC slit versus the thickness of 
a metallic plate of $d$. The maximum periodic TCSs are observed at a plate thickness of $(0.486+0.5 n) \lambda_{0}$, where $n$ is an integer. Figure $2 b-d$ shows the TCSs in terms of thickness of the metallic plate when the permittivities of a metal $\varepsilon_{r, m}$ are $-2000,-100$, and -5 , respectively. The maximum periodic TCSs in Figure $2 b-d$ are also observed at specific thicknesses of the metallic plate. The value of the maximum TCS for the PEC case in Figure 2a is found to be about 0.3183, which is the same as a known theoretical value [27]. By contrast, the maximum TCSs in Figure $2 b-d$ do not reach the theoretical TCS value, because the incident wave from Figure 1 is slightly absorbed into the metallic plate. This could be understood by the fact that a lower metallic permittivity causes greater incident wave absorption into the metallic region [9]. From Equation (4), the transmittance $\tau$ is obtained as described in the inset of Figure 2a. While the pattern is the same as the TCS, numerical values and transmitting units are different, as represented in Equation (4).
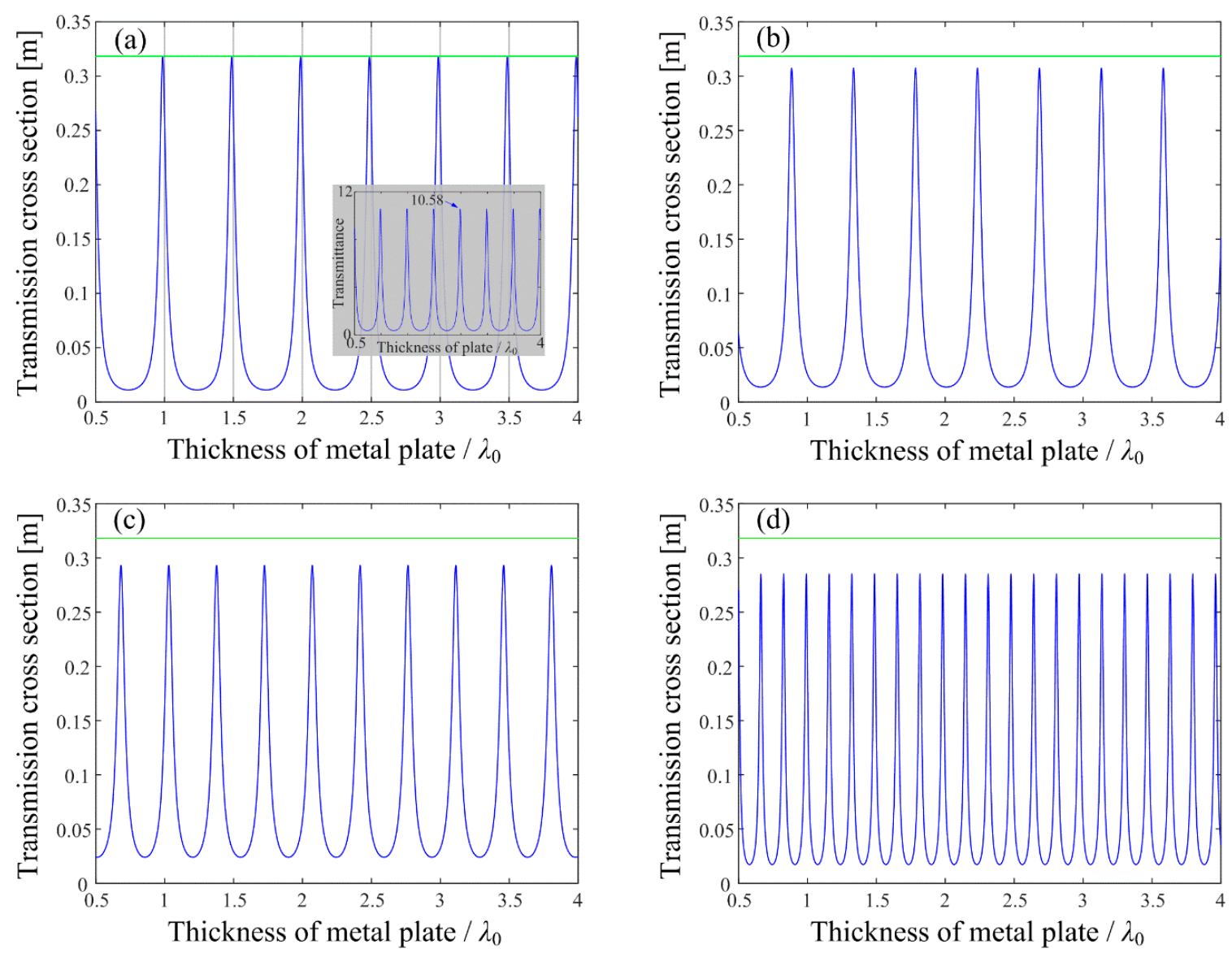

Figure 2. Transmission cross-sections (TCSs) for various permittivities: (a) metal which is a perfect electric conductor (PEC); (b) $\varepsilon_{r}=-2000 ;$ (c) $\varepsilon_{r}=-100$; and (d) $\varepsilon_{r}=-5$. The transmittance for the PEC case is shown in the inset of (a).

Guide wavelengths are helpful to understand resonant transmission characteristics and can be solved from Equations (1) and (2). The guide wavelength $\lambda_{g}$ for each MIM waveguide in Figure 1 is listed in Table 1 . The values of $\lambda_{g}$ for cases of low permittivity are relatively great, while the $\lambda_{g}$ values for high permittivity cases are smaller. Meanwhile, the intervals of TCSs peaks were obtained as $0.5 \lambda_{0}$ and $0.1650 \lambda_{0}$ for the PEC and the metallic permittivity of -5 , respectively, as found in Figure 2 . These intervals, computed by the MMT, are exactly the same as $1 /\left(2 \lambda_{g}\right)$, as listed in the last column of Table 1 . Therefore, resonant transmission phenomena were systematically solved as a function of metallic permittivity, and their periodicity can be easily understood. 
Table 1. Guide wavelengths with respect to metallic permittivities and computed maximum TCS intervals.

\begin{tabular}{ccc}
\hline Permittivities in Metal & Guide Wavelength $\left(\boldsymbol{\Lambda}_{\boldsymbol{G}}\right)$ & Maximum TCS Interval \\
\hline PEC & $1.0 \lambda_{0}$ & $0.5 \lambda_{0}$ \\
$\varepsilon_{r}=-2000$ & $1.1124 \lambda_{0}$ & $0.4495 \lambda_{0}$ \\
$\varepsilon_{r}=-100$ & $1.4406 \lambda_{0}$ & $0.3471 \lambda_{0}$ \\
$\varepsilon_{r}=-5$ & $3.0307 \lambda_{0}$ & $0.1650 \lambda_{0}$ \\
\hline
\end{tabular}

\section{Transmittance through a Subwavelength Slit on a Real Metallic Plate}

In Section 2, transmittance results were obtained, when a metal was a PEC or lossless conductor having a finite conductivity, and transmittances for lossy metals are discussed in this section. Figure 1 is again adopted for the geometry, but the conductor is changed into a realistic metal characterized by a finite conductivity with loss, which depends on the function of $\mathrm{THz}$ frequencies. The relative permittivity of a real metal has not only a real part, but also an imaginary part considering metallic loss. The metal we deal with is silver $(\mathrm{Ag})$, and its relative permittivity is $-14.88-j 0.39$ when $\lambda_{0}=582.08 \mathrm{~nm}$ [28]. The complex numbered $\varepsilon_{r, m}$ can be inserted into the dispersion equation known as Equation (1). The corresponding transverse propagation constants $\left(\kappa_{m, n}\right.$ and $\left.\kappa_{i, n}\right)$ and propagation constant $\left(k_{z, n}\right)$, which are all complex numbers, can then be easily solved. The reflection and transmission coefficients at each boundary can be subsequently calculated by the MMT, obtaining the overall transmission results [9-12].

The transmittances are represented by Figure $3 a, b$, when the gap widths $2 g$ become 4 and $8 \mathrm{~nm}$, respectively. The transmittances are investigated by gradually reducing, and finally eliminating, the imaginary part of $\varepsilon_{r, m}$, even if the actual relative permittivity at $\lambda_{0}=582.08 \mathrm{~nm}$ is $14.88-j 0.39$ [28]. Figure 3 a shows the transmittances through a subwavelength slit versus the thickness of a metallic plate when the gap width $2 g$ is $4 \mathrm{~nm}\left(\sim 0.0069 \lambda_{0}\right)$. With no imaginary part in $\varepsilon_{r, m}$, periodically obtained peak transmittances are maintained although the thickness of the plate increases, because there is no metallic loss, producing the same result trend shown in Figure 2. By contrast, resonant peak transmittances are gradually reduced as the imaginary part of $\varepsilon_{r, m}$ increases. This can be understood that increasing the plate thickness makes electromagnetic waves to pass through the metal over a longer distance, causing more power to be lost due to metallic loss. As the imaginary part of the relative permittivity increases up to $-j 0.39$, attenuation phenomena can be observed clearly.
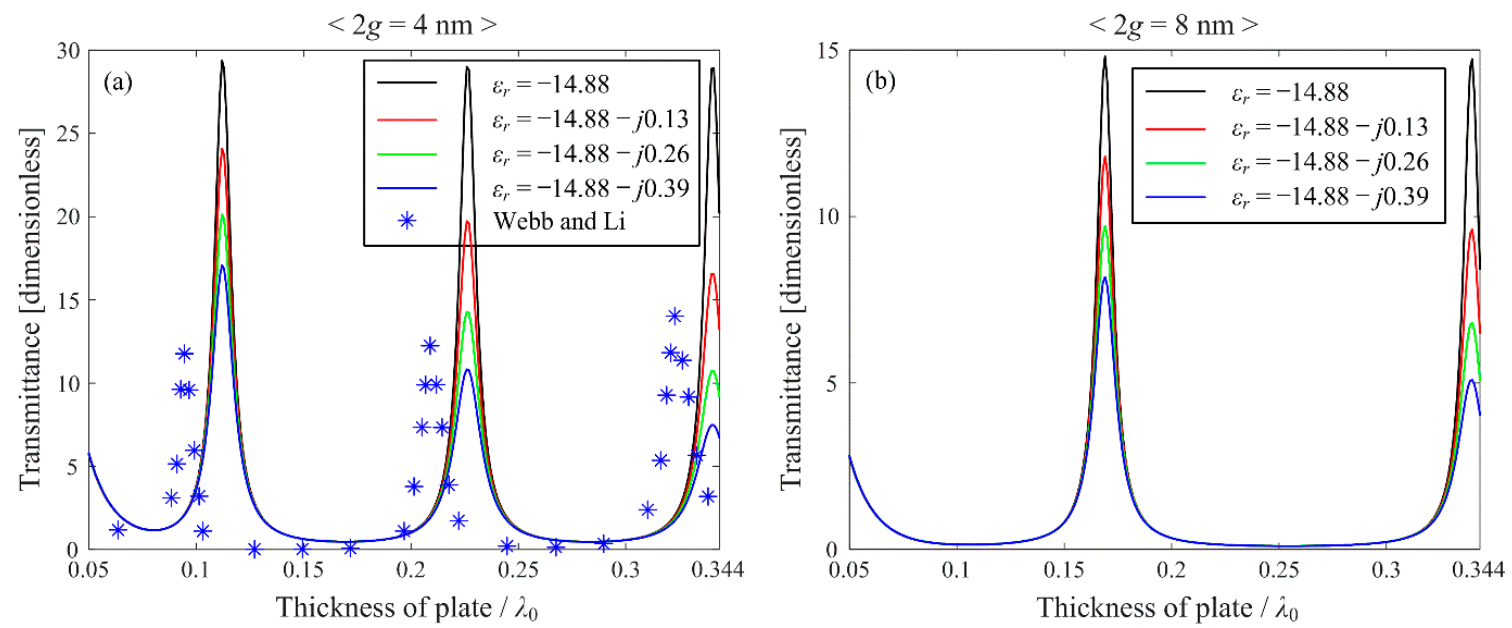

Figure 3. Transmittance as a function of Ag plate thickness: (a) $2 g=4 \mathrm{~nm}$; (b) $2 g=8 \mathrm{~nm}$. The blue asterisks are remarked from [29], when $\varepsilon_{r, m}=-14.88-j 0.39$.

Figure $3 \mathrm{~b}$ shows the transmittances by varying the relative permittivities, when the gap width $2 g$ becomes $8 \mathrm{~nm}\left(0.0137 \lambda_{0}\right)$. The resonant plate thicknesses are maintained despite increased imaginary parts of the permittivities, and the peak transmittances are gradually reduced as the imaginary part 
of $\varepsilon_{r, m}$ increases. However, the interval, at which resonant peaks occur, is increased compared to the interval in Figure 3a. This can be understood using Table 2, which shows the guide wavelengths as functions of the gap width $2 g$ and the relative permittivity of Ag. From each value, the real part of the guide wavelength represents the interval of the periodic transmission peaks, and the imaginary part stands for the attenuation of their peaks. As the real part of the guide wavelength increases, the interval of the transmission peaks decreases, as shown in Figure 3, illustrating the same principle as Table 1. Consequently, as the imaginary part of the guide wavelength increases, the attenuation of the transmission peaks also increases. When the relative permittivity has only a real part, constant maximum transmission peaks in Figure $3 \mathrm{a}, \mathrm{b}$ are found. The maximum transmission peaks are obtained as 29.23 and 14.55, when the gap widths are 4 and $8 \mathrm{~nm}$, respectively. It is important to understand the meaning of these values. First, 29.23 times the incident power density for the gap width of $4 \mathrm{~nm}$ [Figure S1] is transmitted into the opposite (right-hand) region in Figure 1. The same applies to 14.55 times the incident power density for the gap width of $8 \mathrm{~nm}$ [Figure S2] in Figure 3b. Whenever the resonant conditions for both cases are satisfied, the constant width $(\mathrm{m})$ times the incident power density $(\mathrm{W} / \mathrm{m})$ is transmitted into the opposite region, regardless of the width of the subwavelength slit. In other words, the incident power density having a constant width, which is much wider than that of the subwavelength slit, is transmitted into the right side of Figure 1 regardless of the gap width $2 g$, when the resonant condition (thickness of the plate) is satisfied.

Table 2. Guide wavelengths $\left(\lambda_{g}\right)$ with respect to the Ag permittivity and modified permittivities, when the gap width $2 g$ of 4 and $8 \mathrm{~nm}$ are used. $\left(\varepsilon_{r}=-14.88-j 0.39\right.$ at $\lambda_{0}=582.08 \mathrm{~nm}$ [28]).

\begin{tabular}{ccc}
\hline Permittivities of Metal & $\mathbf{2 g}=\mathbf{4} \mathbf{~ n m}$ & $\mathbf{2 g}=\mathbf{8} \mathbf{~ m}$ \\
\hline$\varepsilon_{r}=-14.88$ & 4.3822 & 2.9284 \\
$\varepsilon_{r}=-14.88-j 0.13$ & $4.3820-j 0.0194$ & $2.9283-j 0.0093$ \\
$\varepsilon_{r}=-14.88-j 0.26$ & $4.3815-j 0.0388$ & $2.9281-j 0.0185$ \\
$\varepsilon_{r}=-14.88-j 0.39$ & $4.3805-j 0.0582$ & $2.9277-j 0.0277$ \\
\hline
\end{tabular}

It is necessary to mention the blue asterisks in Figure 3a, which are remarked from [29]. The transmittances versus the thickness of the Ag plate are obtained when Ag is $-14.88-j 0.39$ (at $\lambda_{0}=582.08 \mathrm{~nm}$ ), which is the same relationship as indicated by the blue solid line using the MMT. The overall transmittance levels are similar to each other, and the intervals of the maximum transmittances are also analogous. The maximum transmittances are observed at different resonant thicknesses of the plate, and the difference between [29] and our result is roughly $0.02 \lambda_{0}$. Furthermore, the maximum transmittance markers increase, even though the thickness of the plate increases. This is unnatural, because the electromagnetic wave passing through the slit should be dissipated more as the plate thickness increases. On the contrary, the maximum transmittances by the solid lines of our work gradually decrease and are physically agreeable. Therefore, the transmittances of our work are more convincing than those of [29].

\section{Orthogonality and Discussions}

When the metal of an MIM waveguide is a PEC, field profiles of entire modes (including a transverse electromagnetic mode) can be expressed easily, and the sinusoidal modal variations are found in the slit of the waveguide, while field profiles cannot be expressed in the PEC region. The orthogonal property is completely satisfied, because inner products among the sinusoidal harmonics in the silt are perfectly orthogonal. (In this work, only cosine functions are considered, because only a normally incident wave is dealt with.) The $n$-th modal electromagnetic fields of the parallel plate waveguide in Figure 4a can be expressed as follows:

$$
H_{y}^{n}=\cos \left(\gamma_{n} x\right) e^{-k_{n} z},
$$




$$
\begin{aligned}
E_{x}^{n} & =-\frac{1}{j \omega \varepsilon} \frac{\partial H_{y}^{n}}{\partial z}, \\
\gamma_{n}=\frac{2 n \pi}{2 g} \quad \text { and } \quad k_{n} & =\sqrt{\gamma_{n}^{2}-\omega^{2} \mu \varepsilon}, n=0,1,2, \cdots .
\end{aligned}
$$

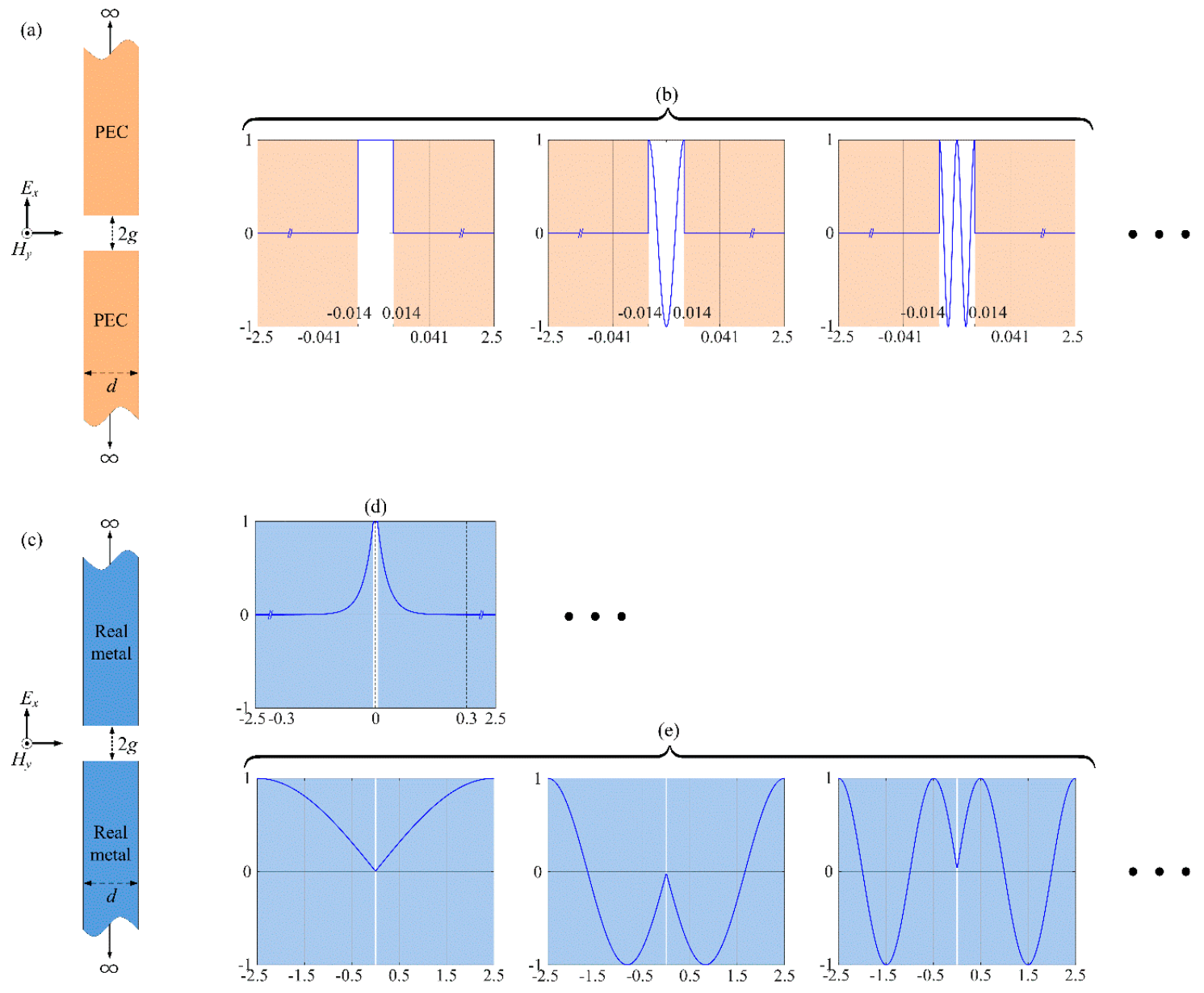

Figure 4. Geometries of different metal-insulator-metal (MIM) waveguides for gap width $2 g=8 \mathrm{~nm}$, when a transverse magnetic (TM) polarized electromagnetic wave is normally incident: (a) a PEC as the conducting material in the MIM waveguide and (b) the corresponding field profiles of the guided modes (transverse electromagnetic (TEM), $\mathrm{TM}_{2}$, and $\mathrm{TM}_{4}$ modes); (c) a practical metal as the conducting material in the MIM waveguide, such as silver and the corresponding field profiles for a point spectrum (PS) (d) and a discretized continuous spectrum (DCS) (e). Note that different scales are shown within the periods of $(\mathbf{b}, \mathbf{d})$.

From the geometry in Figure 4a, the field profiles of the first three even modes are described in Figure $4 \mathrm{~b}$. Note that the scales are different for the slit (white) and metal (light orange) regions. By applying higher-order modes and the first three modes into the MMT, the transmittances are solved. The orthogonal properties among the modes are calculated and confirmed from the following equations [30]:

$$
\begin{gathered}
\frac{1}{\eta_{0}} \frac{k_{0}}{k_{n}} \frac{1}{2 g} \int_{-g}^{g}\left[E_{x}^{n}\left(H_{y}^{m}\right)^{*}\right] d x=\delta_{n m}, \\
\delta_{n m}=\left\{\begin{array}{c}
1, n=m \\
0, n \neq m
\end{array} .\right.
\end{gathered}
$$


From Equations (8) and (9), the calculated result is 1 when the modes are the same, and 0 when the two modes are different. Therefore, the orthogonal property is completely satisfied, when the metal of the MIM waveguide is a PEC. When the metal has a finite conductivity, the geometry of the single slit can be depicted by Figure $4 \mathrm{c}$ with the blue-colored metallic region and the corresponding field profiles shown in Figure 4d,e. The equations for the field profiles are represented as [9]:

$$
H_{y}^{n}=\left\{\begin{array}{c}
\frac{\cosh \left(\kappa_{i, n} x\right)}{\cosh \left(\kappa_{i, n} g\right)} e^{-k_{z, n} z}, 0<x<g \\
e^{-\kappa_{m, n}(x-g)} e^{-k_{z, n} z}, g<x<\infty
\end{array} .\right.
$$

By inserting $\varepsilon_{r, m}$ into Equations (1) and (2), the $n$-th transverse propagation constants ( $\kappa_{i, n}$ and $\left.\kappa_{m, n}\right)$ and the $n$-th propagation constant $\left(k_{z, n}\right)$ are easily obtained. The field profiles can then be obtained from Equation (10) and are described in Figure 4d,e, which stand for the field profiles of a point spectrum (PS) and a discretized continuous spectrum (DCS) [9], respectively. The PS and DCS correspond to the guided and radiation modes in the dielectric slab waveguide, respectively. The PS can describe the sinusoidal electromagnetic field patterns mainly in the "insulator", while the DCS depicts the sinusoidal field patterns mainly in the "metal" region $[9,10]$. The right-hand side of Equation (8), for a metal having a finite conductivity, is not solved exactly as 1 or 0 , which indicates the orthogonality is not perfectly satisfied, but the modes are applied into the MMT and the corresponding transmittances are solved.

The field profiles of the PS and the DCS are described in Figure 4d,e, respectively, where the gap width $2 g$ is $4 \mathrm{~nm}$ and $\varepsilon_{r, m}$ is -14.88 . Figure $4 \mathrm{~d}$ shows the field profiles for the first mode of the PS and may correspond to the first field profile of Figure $4 \mathrm{~b}$. The field shape in the white insulator region is almost maintained; however, the field shape in the sky-blue metal region is different. The field at the boundary between the insulator and the metal penetrates into the metal region, because the metal is no longer a PEC. Figure 4e describes the field profiles of the first, second, and third modes of the DCS. The field profiles represent sinusoidal harmonics in the metal region, and various field patterns in the metal region can be described by superposition of the modes in the DCS.

The numbers of the PS and the DCS used in the MMT formulation are 1 and 50, respectively. Only one mode of the PS is applied to solve the transmittance, because the slit is too narrow compared to the wavelength. That being said, a sufficient number of modes of the DCS should be applied, given the wideness of the metal region compared to that of the slit region [12]. The sufficient number of DCS modes is obtained as follows: We start to apply 5 trial DCS modes and investigate the reflection and transmission coefficients. Computing the coefficients by increasing the number of the DCS modes to 5 are repeated, and then the coefficients are arranged with respect to the number of DCS modes. Once the convergence is found, the updated number of the DCS modes are obtained.

From the viewpoint of orthogonality, the PS and the DCS are clearly independent, because the field patterns of both are formed in different regions; therefore, the result of Equations (8) and (9) between the two approaches 0 . However, the orthogonal properties among 50 DCS modes require investigation, since the metal is not a PEC. Figure 5 indicates in Figure 5 a the results of Equations (8) and (9) of the first 10 DCS modes when $\varepsilon_{r, m}$ of the MIM waveguide is -14.88 . Each cell represents the result of Equations (8) and (9) between two modes. What we need to pay attention to Equations (8) and (9) for the MIM waveguide is the interval of integration. The interval should cover the entire waveguide junction including the metal region as well as the gap region of $2 g$, while the interval of integration $2 g$ in Equations (8) and (9) is still valid in the case of the PEC waveguide. The diagonal components approach approximately 1 when the DCS modes used in Equations (8) and (9) are identical, while the off-diagonal results go to approximately 0 when the modes differ. For the first 10 modes of the DCS, the values from Equations (8) and (9) are within [1.0082, 1.0111] for the same modes, whereas the values are within $\left[4.878 \times 10^{-5}, 0.00340\right]$ for different modes. These computed results are still effective up to the next 40 DCS modes. 
(a)

\begin{tabular}{|c|r|r|r|r|r|r|r|r|r|r|}
\hline DCS & \multicolumn{1}{|c|}{ 1st mode } & 2nd mode & 3rd mode & 4th mode & 5th mode & 6th mode & 7th mode & 8th mode & 9th mode & 10th mode \\
\hline 1st mode & 1.01105 & 0.00005 & 0.00008 & 0.00011 & 0.00014 & 0.00016 & 0.00019 & 0.00021 & 0.00023 & 0.00024 \\
\hline 2nd mode & 0.00005 & 1.01096 & 0.00024 & 0.00033 & 0.00041 & 0.00049 & 0.00056 & 0.00062 & 0.00068 & 0.00073 \\
\hline 3rd mode & 0.00008 & 0.00024 & 1.01078 & 0.00054 & 0.00068 & 0.00081 & 0.00092 & 0.00103 & 0.00112 & 0.00120 \\
\hline 4th mode & 0.00011 & 0.00033 & 0.00054 & 1.01053 & 0.00093 & 0.00111 & 0.00127 & 0.00141 & 0.00154 & 0.00165 \\
\hline 5th mode & 0.00014 & 0.00041 & 0.00068 & 0.00093 & 1.01022 & 0.00139 & 0.00159 & 0.00177 & 0.00193 & 0.00207 \\
\hline 6th mode & 0.00016 & 0.00049 & 0.00081 & 0.00111 & 0.00139 & 1.00986 & 0.00189 & 0.00211 & 0.00229 & 0.00246 \\
\hline 7th mode & 0.00019 & 0.00056 & 0.00092 & 0.00127 & 0.00159 & 0.00189 & 1.00946 & 0.00241 & 0.00262 & 0.00281 \\
\hline 8th mode & 0.00021 & 0.00062 & 0.00103 & 0.00141 & 0.00177 & 0.00211 & 0.00241 & 1.00904 & 0.00292 & 0.00312 \\
\hline 9th mode & 0.00023 & 0.00068 & 0.00112 & 0.00154 & 0.00193 & 0.00229 & 0.00262 & 0.00292 & 1.00861 & 0.00340 \\
\hline 10th mode & 0.00024 & 0.00073 & 0.00120 & 0.00165 & 0.00207 & 0.00246 & 0.00281 & 0.00312 & 0.00340 & 1.00817 \\
\hline
\end{tabular}

(b)

\begin{tabular}{|c|r|r|r|r|r|r|r|r|r|r|}
\hline DCS & 1st mode & 2nd mode & 3rd mode & 4th mode & 5th mode & 6th mode & 7th mode & 8th mode & 9th mode & 10th mode \\
\hline 1st mode & 1.00851 & 0.00005 & 0.00008 & 0.00011 & 0.00014 & 0.00016 & 0.00019 & 0.00021 & 0.00023 & 0.00024 \\
\hline 2nd mode & 0.00005 & 1.00845 & 0.00024 & 0.00033 & 0.00041 & 0.00049 & 0.00056 & 0.00062 & 0.00068 & 0.00073 \\
\hline 3rd mode & 0.00008 & 0.00024 & 1.00833 & 0.00055 & 0.00068 & 0.00081 & 0.00092 & 0.00103 & 0.00112 & 0.00120 \\
\hline 4th mode & 0.00011 & 0.00033 & 0.00055 & 1.00816 & 0.00094 & 0.00111 & 0.00127 & 0.00141 & 0.00154 & 0.00165 \\
\hline 5th mode & 0.00014 & 0.00041 & 0.00068 & 0.00094 & 1.00794 & 0.00141 & 0.00160 & 0.00177 & 0.00193 & 0.00207 \\
\hline 6th mode & 0.00016 & 0.00049 & 0.00081 & 0.00111 & 0.00141 & 1.00768 & 0.00191 & 0.00211 & 0.00229 & 0.00246 \\
\hline 7th mode & 0.00019 & 0.00056 & 0.00092 & 0.00127 & 0.00160 & 0.00191 & 1.00740 & 0.00243 & 0.00263 & 0.00281 \\
\hline 8th mode & 0.00021 & 0.00062 & 0.00103 & 0.00141 & 0.00177 & 0.00211 & 0.00243 & 1.00709 & 0.00294 & 0.00313 \\
\hline 9th mode & 0.00023 & 0.00068 & 0.00112 & 0.00154 & 0.00193 & 0.00229 & 0.00263 & 0.00294 & 1.00676 & 0.00343 \\
\hline 10th mode & 0.00024 & 0.00073 & 0.00120 & 0.00165 & 0.00207 & 0.00246 & 0.00281 & 0.00313 & 0.00343 & 1.00643 \\
\hline
\end{tabular}

Figure 5. Orthogonal relationships among the first ten DCS modes for gap width $2 g=4 \mathrm{~nm}$ : (a) $\varepsilon_{r}=-14.88$ and (b) $\varepsilon_{r}=-14.88-j 0.39$. The diagonal values are obtained from Equations (8) and (9) when $n=m(n=1,2, \ldots, 10)$, and the off-diagonal values are obtained from Equations (8) and (9) when $n \neq m$. Note: Cells highlighted in yellow indicate that the numerical values of the diagonal cells approach 1 .

The orthogonality changes slightly but remains almost constant despite the inclusion of an imaginary part in the permittivity of the metal. The field profiles of the PS and the DCS in Figure 4d,e change slightly, although $\varepsilon_{r, m}$ becomes $-14.88-j 0.39$. Figure $5 \mathrm{~b}$ shows the orthogonal results of Equations (8) and (9) among the first 10 DCS modes, when $\varepsilon_{r, m}$ is $-14.88-j 0.39$. The solved results are very similar to Figure $5 \mathrm{a}$, even though the imaginary part of the permittivity is included. For the first 10 modes of the DCS, the values from Equations (8) and (9) are within [1.0064, 1.0085] for the identical modes, while the values are within $\left[4.945 \times 10^{-5}, 0.00343\right]$ for different modes. Therefore, the orthogonal relationships are almost the same when $\varepsilon_{r, m}$ is -14.88 , where an orthogonal set is almost remains. As the relationships of the next 40 DCS modes are considered, the orthogonal properties are maintained. Although the orthogonality for the real metal case is not completely satisfied, it is not enough to affect the accuracy of transmittance. Despite this incomplete orthogonality, the transmission characteristics are mainly determined by the fundamental mode, so that the accurate results can still be maintained. The field profiles by higher-order modes change more, but the higher-order modes do not significantly affect the slit transmission.

The guide wavelengths $\lambda_{g}$ of the DCS, as well as that of the PS modes, are also investigated to allow the transmission fundamentals to be understood. The $\lambda_{g}$ value of the first PS mode is 4.382, when the gap width $2 g$ is $4 \mathrm{~nm}$ and the permittivity of $\mathrm{Ag} \varepsilon_{r, m}$ is -14.88 . By contrast, the $\lambda_{g}$ value of the first DCS mode is purely imaginary and roughly $-j 3.859$ under the same conditions. This is significantly greater than the imaginary parts of the PS listed in Table 2, demonstrating the $\lambda_{g}$ value of the DCS attenuates significantly and cannot contribute to the transmission. Therefore, the DCS modes do not contribute to the transmission but perform a complete orthogonal set for the MMT [10].

\section{Conclusions}

In this paper, resonant transmission phenomena through a single metallic subwavelength slit were investigated, when the permittivity of $\mathrm{Ag}$ was changed with varying frequencies. The concept of the MIM waveguide was adopted for the single metallic slit, and the complex permittivity of real metal was considered regarding the MIM waveguide. The eigenvalues of the MIM waveguide 
were then applied to the MMT, and the transmittances were solved. The periodic transmission characteristics versus the thicknesses of the plate were obtained as a function of metallic permittivity, and their periodicities were easily understood using each guide wavelength. The imaginary parts of the permittivities for real metals were subsequently included to obtain corresponding transmittance characteristics. Similarly, the periodicities and attenuations were understood, thanks to the guide wavelengths of the MIM geometry. Finally, the orthogonal properties among the eigenvalues of the DCS were investigated for given lossy and lossless real metals in order to investigate the validity of their MMT formulations. For all cases, the orthogonal relationships among the same modes were obtained at approximately one, while the orthogonal relationships among different modes were obtained at no more than 0.0034 . Therefore, the MMT formulations, even for the lossy cases, were deemed reasonable to solve transmittances.

Supplementary Materials: The following are available online at http://www.mdpi.com/2076-3417/10/2/660/s1, Figure S1: Physical meaning of resonant transmission when $2 g=4 \mathrm{~nm}$, Figure S2: Physical meaning of resonant transmission when $2 g=8 \mathrm{~nm}$.

Author Contributions: Conceptualization, J.-E.P. and Y.-K.C.; methodology, J.-E.P.; software, J.-E.P.; validation, J.-E.P.; formal analysis, J.-E.P. and Y.-K.C.; investigation, J.-E.P. and Y.-K.C.; writing of the original draft preparation, J.-E.P. and Y.-K.C.; writing of review and editing, J.-E.P. and H.C.; visualization, J.-E.P.; supervision, H.C.; project administration, Y.-K.C.; funding acquisition, H.C. All authors have read and agreed to the published version of the manuscript.

Funding: This research was funded in part by the Nuclear Safety Research Program through the Korea Foundation of Nuclear Safety (KoFONS) using the financial resource granted by the Nuclear Safety and Security Commission (NSSC) of the Republic of Korea (No. 1805006) and in part by the National Research Foundation of Korea (NRF) funded by the Ministry of Education (Nos. 2013R1A6A3A03022194, 2015R1A6A1A03031833, and 2017R1D1A1B04031890).

Conflicts of Interest: The authors declare no conflicts of interest.

\section{References}

1. Ebbesen, T.W.; Lezec, H.J.; Ghaemi, H.F.; Thio, T.; Wolff, P.A. Extraordinary optical transmission through sub-wavelength hole arrays. Nature 1998, 391, 667-699. [CrossRef]

2. Guo, J.; Leong, H. Mode splitting of surface plasmon resonance in super period metal nanohole array gratings. Appl. Phys. Lett. 2012, 101. [CrossRef]

3. Azad, A.K.; O'Hara, J.F.; Singh, R.; Chen, H.-T.; Taylor, A.J. A review of terahertz plasmonics in subwavelength holes on conducting films. IEEE J. Sel. Top. Quantum Electron. 2012, 19. [CrossRef]

4. Lee, D.; Kim, D.-S. Light scattering of rectangular slot antennas: Parallel magnetic vector vs perpendicular electric vector. Sci. Rep. 2016, 6. [CrossRef] [PubMed]

5. Auckland, D.T.; Harrington, R.F. Electromagnetic transmission through a filled slit in a conducting plane of finite thickness, TC case. IEEE Trans. Microw. Theory Tech. 1978, 26, 499-505. [CrossRef]

6. Harrington, R.F.; Auckland, D.T. Electromagnetic transmission through narrow slots in thick conducting screen. IEEE Trans. Antennas Propag. 1980, 28, 616-622. [CrossRef]

7. Sturman, B.; Podivilov, E.; Gorkunov, M. Eigenmodes for metal-dielectric light-transmitting nanostructures. Phys. Rev. B 2007, 76. [CrossRef]

8. Sturman, B.; Podivilov, E.; Gorkunov, M. Theory of extraordinary light transmission through arrays of subwavelength slits. Phys. Rev. B 2008, 77. [CrossRef]

9. Kocabas, S.E.; Veronis, G.H.; Miller, D.A.B.; Fan, S. Modal analysis and coupling in metal-insulator-metal waveguides. Phys. Rev. B 2009, 79. [CrossRef]

10. Park, J.E.; Teixeira, F.L.; Borges, B.H. Analysis of deep-subwavelength Au and Ag slit transmittances at terahertz frequencies. J. Opt. Soc. Am. B 2016, 33, 1355-1364. [CrossRef]

11. Hur, J.; Choo, H.; Park, J.E. Modal analysis of point and discretized continuous spectra for metal-insulator-metal waveguides in the terahertz region. J. Electr. Eng. Technol. 2018, 13, 1644-1654.

12. Yoo, S.; Park, J.E.; Choo, H. Resonant transmission through periodic subwavelength real metal slits in the terahertz range. IEICE Electron. Expr. 2018, 15, 1-11. [CrossRef] 
13. Wang, S.; Teixeira, F.L. Lattice models for large-scale simulations of coherent wave scattering. Phys. Rev. E 2004, 69. [CrossRef] [PubMed]

14. Texeira, F.L. Time-domain finite-difference and finite-element methods for Maxwell equations in complex media. IEEE Trans. Antennas Propag. 2008, 56, 2150-2166. [CrossRef]

15. Rim, J.-W.; Koh, I.-S. SAR image generation of ocean surface using time-divided velocity bunching model. J. Electromagn. Eng. Sci. 2019, 19, 82-88. [CrossRef]

16. Seo, S.M. An IE-FFT algorithm to analyze PEC objects for MFIE formulation. J. Electromagn. Eng. Sci. 2019, 19, 6-12. [CrossRef]

17. Tareki, A.M.; Lindquist, R.G.; Kim, W.; Heimbeck, M.S.; Guo, J. Terahertz transparent electrode using tripod metal aperture array. IEEE Trans. Terahertz Sci. Technol. 2016, 7, 80-85. [CrossRef]

18. Zarei, S. A design to tune the frequency in a terahertz filter based on dual layered metallic slit arrays. Photon. Nanostruct. Fundam. Appl. 2019, 34, 5-10. [CrossRef]

19. Dhawan, A.; Gerhold, M.D.; Muth, J.F. Plasmonic structures based on subwavelength apertures for chemical and biological sensing applications. IEEE Sens. J. 2008, 8, 942-950. [CrossRef]

20. Larson, S.; Carlson, D.; Ai, B.; Zhao, Y. The extraordinary optical transmission and sensing properties of Ag/Ti composite nanohole arrays. Phys. Chem. Phys. 2019, 21, 3771-3780. [CrossRef]

21. Alfalou, A.; Brosseau, C. Dual encryption scheme of images using polarized light. Opt. Lett. 2010, 35, 2185-2187. [CrossRef] [PubMed]

22. Wang, Y.; Tong, Y.; Zhang, X. Transmission properties of terahertz waves through asymmetric rectangular aperture arrays on carbon nanotube films. AIP Adv. 2016, 6. [CrossRef]

23. Nakata, Y.; Urade, Y.; Okimura, K.; Nakanishi, T.; Miyamaru, F.; Takeda, M.W.; Kitano, M. Anisotropic Babinet-invertible metasurfaces to realize transmission reflection switching for orthogonal polarizations of light. Phys. Rev. Appl. 2016, 6. [CrossRef]

24. Pelzman, C.; Cho, S.-Y. Multispectral and polarimetric photodetection using a plasmonic metasurface. J. Appl. Phys. 2018, 123. [CrossRef]

25. Lee, G.; Maeng, I.; Kang, C.; Oh, M.-K.; Kee, C.-S. Strong polarization-dependent terahertz modulation of aligned Ag nanowires on Si substrate. Opt. Express 2018, 26, 13677-13685. [CrossRef]

26. Pattanayak, A.; Rana, G.; Jain, R.; Bhattacharya, A.; Duttagupta, S.P.; Gandhi, P.S.; Achanta, V.G.; Prabhu, S.S. Resonant $\mathrm{THz}$ transmission through asymmetric aperture array with polarization controlled resonant peaks and Q-factors. J. Appl. Phys. 2019, 126. [CrossRef]

27. Verslegers, L.; Yu, Z.; Catrysse, P.B.; Fan, S. Temporal coupled-mode theory for resonant apertures. J. Opt. Soc. Am. B 2010, 27, 1947-1956. [CrossRef]

28. Johnson, P.B.; Christy, R.W. Optical constants of the noble metals. Phys. Rev. B 1972, 6, 4370-4379. [CrossRef]

29. Webb, K.J.; Li, J. Analysis of transmission through small apertures in conducting films. Phys. Rev. B 2006, 73. [CrossRef]

30. Harrington, R.F. Time-Harmonic Electromagnetic Fields; McGraw-Hill College: New York, NY, USA, $1961 ;$ p. 432.

(C) 2020 by the authors. Licensee MDPI, Basel, Switzerland. This article is an open access article distributed under the terms and conditions of the Creative Commons Attribution (CC BY) license (http://creativecommons.org/licenses/by/4.0/). 